Planetary Systems in the Universe - Observation, Formation and Evolution

Proceedings IAU Symposium No. 202, (C)2004 IAU

Alan Penny, Pawel Artymowicz, Anne-Marie Lagrange, \& Sara Russell, eds.

\title{
A Model of Stochastic Collisions as the Cause of Clumps in Debris Disks
}

\author{
M. C. Wyatt, W. R. F. Dent \\ UKATC, Royal Observatory, Edinburgh, EH9 3HJ, UK \\ J. S. Greaves, W. S. Holland \\ JAC, 660 North A'ohoku Place, Hilo, HI 96720, USA
}

\begin{abstract}
We present a heuristic model for the collisional evolution of material in a debris disk. This is used to consider the probability that the $2-3 \%$ brightness clump observed in the sub-mm Fomalhaut disk is caused by stochastic collisions between large planetesimals. While this simple model finds that the probability that the clump is caused by collisions is low (about 1 in 80,000), a more detailed model is required to ascertain its true likelihood.
\end{abstract}

\section{Introduction}

Images of the thermal emission from the dust disks that surround some main sequence stars show that the dust is confined to a narrow ring at a location analagous to the Kuiper belt in the solar system. The short lifetime of this dust, due to both mutual collisions and radiation forces, implies that it must be continually replenished. It is thought that the dust disk is fed by the collisional grinding down of a population of larger planetesimals which may have formed during the system's planetary formation phase (Backman \& Paresce 1993). The images also show that the dust rings are neither smooth nor symmetrical: e.g., recent sub-mm images of the Fomalhaut disk show that embedded within this there may be a clump containing $2-3 \%$ of the total disk flux (Holland et al., in prep); there are clumps in the $\epsilon$ Eridani disk, the largest of which contains 6-7\% of the disk flux (Greaves et al. 1998); and there is a $5 \%$ brightness asymmetry in the structure of the HR4796 disk (Telesco et al. 2000), which could be accounted for by a clump embedded in the NE lobe that contains $\sim 0.5 \%$ of the disk flux.

It is often speculated that the absence of dust close to the star indicates that a planetary system may have formed there. If this was the case, then the gravitational perturbations of the planets to the orbits of the planetesimals in the disk could cause the observed dust disk to contain both offset and warp asymmetries (Wyatt et al. 1999) as well as resonant clumps (Ozernoy et al. 2000). However, the interpretation of observations of such disk structures in terms of planets should be treated with caution, since there may be alternative explanations for the origin of these structures. Here we explore the possibility that stochastic collisions between a disk's largest planetesimals could cause its structure to be clumpy. 


\section{Stochastic Collision Model}

In order to work out how many clumps of a given size we can expect to observe in a disk at any given time due to collisional processes, we need to consider the following questions: 1) how often do collisions between planetesimals in the disk occur, 2) how much dust is produced in these collisions, and 3) how long does the resulting dust clump last? Simple assumptions about the disk and the outcome of collisions are used here to provide answers to these questions. All notation is the same as that used in Wyatt et al. (1999).

1. The collisional lifetime of a planetesimal of size $D$ depends on the disk's size distribution, since it is only collisions with objects larger than $D_{c c}=X_{c c} D$, where $X_{c c}$ is determined by the planetesimal's impact strength, that have enough energy to be destructive. Here we assume that this distribution follows a power law described by the exponent $q_{d}$ (where number of particles per unit mass is $\propto m^{-q_{d}}$ ) for particles larger than $D_{\text {min }_{d}}$; in which case the total cross-sectional area in the disk, $\sigma_{\text {tot }}$, defines $D_{\text {max }_{d}}$, the size of the largest planetesimal in this distribution, which we assume to be the one there is only one of. The collisional lifetime of planetesimals in such a disk is derived in Wyatt et al. (1999).

2. If the fragments created in the collision also follow a power law, described by $q_{c}$ and $D_{\min _{c}}$, conservation of mass of the parent planetesimal gives the size of the largest fragment, $D_{\max _{c}}$, and the cross-sectional area created in the collision, $\sigma_{c}$. It is useful to express $\sigma_{c}$ in terms of the fractional increase in the cross-sectional area of the disk, $C=\sigma_{c} / \sigma_{t o t}$.

3. The outcome of a collision is to form a clump of dust and debris which follows the orbit of the parent planetesimal. This clump disperses, on a timescale, $t_{c l}$, since the fragments precess around the orbit at different rates depending on the velocity imparted to them in the collision. This velocity will be small if $q_{d}>10 / 6$, since with this distribution a planetesimal is most likely to be broken up by one that only just has enough energy to do so, i.e., by one of size $\sim X_{c c} D$.

Here we also make the following assumptions: $q_{d}=q_{c}=11 / 6$, which is that expected for a steady state collisional cascade; $D_{\text {min }_{d}}=D_{\min _{c}}=10$ $\mu \mathrm{m}$, the approximate radiation pressure blow-out diameter; $X_{c c}=0.1$, which is that assumed for the HR4796 disk by scaling from the asteroid belt (Wyatt et al. 1999); and $t_{c l} / t_{\text {per }}=100$.

Consider a disk with an effective optical depth, $\tau_{\text {eff }}$, a ring radius, $r$, and a ring width, $d r$. Combining 1) and 3), this model predicts that at any one time, the average number of clumps in the disk created by the breakup of planetesimals that are larger than $D$ is given by:

$$
N_{D} \approx 0.37 r d r \tau_{e f f}^{2} D^{-3} .
$$

Including the assumptions about the cross-sectional area created in collisions (2), we find that the average number of clumps we can expect to see each containing more than $C$ times the total disk flux is given by:

$$
N_{C} \approx 33(r d r)^{-0.2} \tau_{e f f}^{0.8} C^{-1.2} .
$$




\section{Application to Fomalhaut}

Applying this model to the Fomalhaut disk, for which $r=100 \mathrm{AU}, d r=40 \mathrm{AU}$, and $\tau_{\text {eff }}=8 \times 10^{-5}$, we find that the disk should contain one clump caused by the breakup of planetesimals larger than $600 \mathrm{~km}$, while clumps from the breakup of smaller bodies are more common, so that they appear as part of the smooth background disk. This means that we can expect to see one clump in the disk with roughly $2 \times 10^{-6}$ times the total disk flux. It is also possible to see brighter clumps; the model predicts that the probability of seeing a clump with more than $2 \%$ of the total disk flux is about 1 in 80,000 .

While this model predicts that it is unlikely that Fomalhaut's sub-mm clump is caused by stochastic collisions, we cannot rule out a collisional origin for the clump at this stage, because the simple assumptions of this preliminary model could be in error. For example, setting $q_{d}=11 / 6$ implies that steady state collisional processes dominate the disk's size distribution, whereas in a young (200 Myr) disk, this distribution may more closely resemble one dominated by planetary formation processes. Also, even in a steady state collisional cascade, a size distribution with $q_{d}=11 / 6$ is not expected to hold down to $D_{\text {min }_{d}}$. The validity of the modelled size distribution in the case of Fomalhaut is certainly questionable, since it predicts that $D_{\text {max }_{d}}=60,000 \mathrm{~km}$, corresponding to $M_{\text {max }_{d}}=30 M_{\oplus}$ for $\rho=2500 \mathrm{~kg} / \mathrm{m}^{3}$. Even if such a massive planetesimal was able to form, its perturbations to the disk would clear a gap around its orbit and impose structure on the disk (Wyatt 1999). Equally, the size distribution of collision fragments may not follow $q_{c}=11 / 6$, and again it is not certain how well such power law distributions can be extrapolated to small sizes. Evidence from the asteroid families implies that $q_{c}$ could be larger than 11/6 (Tanga et al. 1999), implying that we may be underestimating $\sigma_{c}$. Finally, energy conservation considerations imply that 100 orbital periods is only a lower limit to $t_{c l}$, which may actually be much longer. For a more detailed study of the collisional evolution model, the reader is referred to a forthcoming paper by the authors (Wyatt et al., in prep).

\section{References}

Backman, D. E., \& Paresce, F. 1993, in Protostars and Planets III, 1253

Greaves, J. S., et al. 1998, ApJ, 506, L133

Ozernoy, L. M., et al. 2000, ApJ, 537, L147

Tanga, P., et al. 1999, Icarus, 141, 65

Telesco, C. M., et al. 2000, ApJ, 530, 329

Wyatt, M. C. 1999, Ph.D. Thesis, Univ. Florida

Wyatt, M. C., et al. 1999, ApJ, 517, 918 